\title{
THE INFLUENCE BETWEEN INJECTABLE PLATELET-RICH FIBRIN AND PLATELET-RICH PLASMA TOWARDS GINGIVAL FIBROBLAST CELL PROLIFERATION
}

Arifia Anindita Danastri*, Suryono**, Kwartarini Murdiastuti ${ }^{\star *}$

${ }^{*}$ Clinical Dentistry Program, Faculty of Dentistry, Universitas Gadjah Mada, Yogyakarta, Indonesia

${ }^{*}$ Department of Periodontics, Faculty of Dentistry, Universitas Gadjah Mada, Yogyakarta, Indonesia

Correspondence: arifianindita@mail.ugm.ac.id

Keywords:

Gingival fibroblast cell proliferation; Injectable platelet-rich fibrin; Plateletrich plasma

\section{ABSTRACT}

Background: Gingiva is the outermost periodontal tissue that acts as a mechanical and biological barrier to the root of the teeth and alveolar bone. The main cellular elements in the gingiva are fibroblasts. Fibroblast cell proliferation is an important process in tissue regeneration. Growth factors that can stimulate fibroblast cell proliferation can be found in regenerative agents, such as injectable platelet-rich fibrin (i-PRF) and platelet-rich plasma (PRP). The aim of this study was to examine the influence between i-PRF and PRP on the gingival fibroblast cell proliferation in vitro study on primary cell culture.

Method: Gingival fibroblast cell were obtained from primary cell culture derived from healthy gingiva. Ten $\mathrm{mL}$ of peripheral blood were centrifuged for $i-P R F$ and PRP preparation. The samples were divided into three groups: $i-P R F, P R P$, and fibroblast cells without treatment. Cell proliferation were observed at day 1, day 3, day 5 using MTT assay at $550 \mathrm{~nm}$. The data were analyzed by TwoWay ANOVA test, followed by Post Hoc test.

Result: The results showed that the cell proliferation increased from day 1, 3, and 5 in all groups. The absorbance value of the cell proliferation in order from highest to lowest: i-PRF, PRP, and cell control.

Conclusion: $i-P R F$ and PRP increased the gingival fibroblast cell proliferation. $i-P R F$ increased the cell proliferation higher than PRP.

\section{INTRODUCTION}

Periodontal disease includes various chronic inflammatory conditions of the gingiva, alveolar bone, and periodontal ligaments that support teeth. ${ }^{1}$ If untreated, this condition can lead to progressive destruction of the gingival tissue, which can affect its aesthetics and function as a tooth support. ${ }^{2}$ Tissue regeneration is required in the treatment of periodontal disease to allow the damaged or lost tissue to regain its shape and function. Regeneration treatment aims to increase the population of progenitor cells in damaged areas. ${ }^{3}$ There are various types of cells that play a role in tissue regeneration responses: fibroblast cells, which have a vital role in gingival regeneration. ${ }^{4}$

Fibroblasts are the dominant cells in the gingival connective tissue, originating from the mesenchyme and play a major role in the 
development, maintenance and repairment of the gingival connective tissue. ${ }^{5}$ With addition of a proper scaffold, it will be able to support the fibroblast cells adhesion and proliferation, thereby accelerating tissue repair. ${ }^{6}$ Fibroblast cell populations are required for regeneration of stable fibril connections between the gingiva, tooth roots, and periodontal ligaments. Connective tissue regeneration involves a variety of cellular activities driven by fibroblast cell populations. ${ }^{4}$ Cells will migrate, proliferate, and mature into an organized and functional fibrous attachment for regeneration to occur. ${ }^{7}$

The success of migration, proliferation, and maturation of progenitor cells depends on the availability of suitable growth factors and contact with the extracellular matrix. ${ }^{2}$ Growth factors play a role in tissue repair, including cell proliferation, chemotaxis, differentiation, and matrix synthesis by binding to receptors of specific cell surface. Various growth factors that consisting of fibroblast growth factors (FGFs), platelet-derived growth factor (PDGF), insulin-like growth factors (IGFs), transforming growth factors (TGFS), epidermal growth factor (EGF), and vascular endothelial growth factor (VEGF) has important role in periodontal tissue regeneration and wound healing. ${ }^{7}$ Growth factor suspensions concentrated in platelets act as bioactive additive material that was applied locally to induce wound healing. ${ }^{8}$

Platelet-rich plasma (PRP) is a concentrate obtained from blood and centrifuged to eliminate red blood cells, ${ }^{8}$ contains high PDGF which is able to modulate wound healing in tissue. ${ }^{9}$ Platelet-rich plasma -has been widely used for regeneration in the field of Dentistry for 30 years. Preparation of PRP using the anticoagulant bovine thrombin became a concern because it can reduce tissue regeneration ability and create an unstable fibrin matrix. Therefore, a platelet concentrate that does not use an anticoagulant was developed, i.e. platelet-rich fibrin (PRF). ${ }^{10}$

Platelet-rich fibrin (PRF) is a second generation platelet concentrate which is divided into two, solid platelet rich fibrin and liquid platelet rich fibrin. ${ }^{11}$ The development of PRF in a liquid form known as injectable platelet-rich fibrin (i-PRF), that possess similar consistency as PRP, aims to easy application by clinicians to use it as a single ingredient or in combination with various biomaterials. ${ }^{10,12}$ The Low-Speed Centrifugation Concept (LSCC) can increase the number of leucocytes, platelets, and growth factors in i-PRF. ${ }^{13}$ The accumulated release of PDGF-AA, PDGF-AB, $E G F$, and IGF-1 in i-PRF is higher than PRP, whereas the accumulated release of PDGF-BB, TGF- $\beta 1$, and VEGF are found to be higher in PRP. Both of them are able to influence the activity of gingival fibroblasts despite its various different properties. $^{10}$

The research by Miron et al. ${ }^{10}$ showed that gingival fibroblast cell proliferation was higher in PRP, whereas the research by Wang et al. ${ }^{12}$ showed that gingival fibroblast cell proliferation was higher in i-PRF. The results difference of the two studies certainly requires further research studies. This study aims to examine the influence of i-PRF and PRP towards the increase of gingival fibroblasts proliferation in vitro study on primary cell culture.

\section{METHOD}

This study was a purely laboratory experimental with a quantitative design. The samples were gingival fibroblasts from primary cell culture. This study has received approval from the dental ethics commission of the UGM Faculty of Dentistry Yogyakarta Number 00514 / KKEP / FKGUGM / EC / 2020 
A healthy gingiva after crown lengthening treatment were obtained as samples. The excised gingival tissue was washed three times using Phosphate-Buffered Saline (PBS). Furthermore, the gingiva was chopped and placed in a culture plate containing Dulbecco's Modified Eagle's Medium (DMEM) culture medium, added with 10\% FBS supplement, $2 \%$ penicillin-streptomycin, $0.5 \%$ fungi zone, then put in a $\mathrm{CO}_{2}$ incubator at $37^{\circ} \mathrm{C}$. Culture plate was examined daily using a microscope and the culture medium was changed twice a week or every three days. After the fibroblasts reached a $70-80 \%$ confluent state, the cells were separated using EDTA-trypsin $0.25 \%$, then transferred to a culture flask and sub-cultured. This is defined as passage 1. The fibroblast cell culture used in the study came from passage 4. ${ }^{14,15,16}$ Fibroblast cells in DMEM were inserted into 96 well microplate as much as $100 \mu$ with a density of $2.5 \times 10^{3}$ cells/well 24 -hours -prior treatment.

Peripheral blood from the donor was collected as much as $10 \mathrm{ml}$ and put into a sterile plastic tube. The blood sample was then centrifuged at $700 \mathrm{rpm}$ for 3 minutes at room temperature. Injectable platelet-rich fibrin is taken from the yellow-colored top layer over the buffy coat. ${ }^{10}$ For PRP preparation, peripheral blood from the donor was collected as much as $10 \mathrm{ml}$, added with EDTA anticoagulant, then centrifuged at $900 \mathrm{rpm}$ for 5 minutes at room temperature. Then, a second centrifugation was carried out at $2000 \mathrm{rpm}$ for 15 minutes at room temperature. ${ }^{12}$

The samples were divided into three groups with 6 replications. Treatment group I Fibroblasts in DMEM inside the well were added with $100 \mu \mathrm{l}$ PRF. Treatment group II Fibroblasts in DMEM inside the well were added with $100 \mu \mathrm{I}$ PRP which was collected from the centrifuge tube. The control group Fibroblasts in DMEM inside the well were not receive any application.
On day $-1,-3$, and -5 , the medium was added with $20 \mu \mathrm{l}$ MTT each well and then incubated. After 4 hours incubation at $37^{\circ} \mathrm{C}$ in a $5 \% \mathrm{CO}_{2}$ incubator, then $100 \mu \mathrm{l} 10 \%$ SDS solution was added as a stop solution to dissolve the formazan crystals formed. Furthermore, the microplate was re-incubated for 24 hours at $37^{\circ} \mathrm{C}$ in a $5 \% \mathrm{CO}_{2}$ incubator and read with a microplate reader at $550 \mathrm{~nm} \cdot{ }^{17}$ The data on the microplate reader were the absorbance values.

Data from i-PRF, PRP, and control cells groups were analyzed using the IBM SPSS version 24. The data distribution test was performed using the Saphiro-Wilk normality test and the data homogeneity test was performed using the Levene test. Data analysis was performed using Two-Way ANOVA parametric analysis test with a significance level of 0.05 and continued by conducting Post Hoc Least Significant Difference (LSD) analysis to determine the significance of differences between each group.

\section{RESULTS}

Table 1 showed there was an increase in the absorbance value of gingival fibroblast cell proliferation starting from day 3 and day 5 in all groups, however the amount of the increase was different. Respectively, the highest increase was in the i-PRF group, the PRP group, and finally the cell control group.

In this study, a parametric statistical test was conducted using Two-Way ANOVA. Prior to the Two-Way ANOVA test, the normality and homogeneity tests were first carried out. The results were normally distributed and homogeneous.

The type of application group and time of observation affected the absorbance value of gingival fibroblast cell proliferation with a significance value of $0.000 \quad(p<0.05)$. The interaction between two groups (type of application and time observation) also affected the absorbance 
value of gingival fibroblast cell proliferation $(p$ $<0.05)$. Figure 1 showed a pattern of increase in the mean of absorbance value of gingival fibroblast cell proliferation after i-PRF, PRP, and cell control applications on day 1, 3, and 5. The Post Hoc test showed significance between two groups.

Table 1. Mean and Standard Deviation of Absorbance Value of Gingival Fibroblast Cells Proliferation

\begin{tabular}{ccccc}
\hline \multicolumn{5}{c}{ Mean and Standard Deviation of Absorbance Value of Gingival Fibroblast Cells } \\
\hline Group & $\mathrm{n}$ & Day 1 & Day 3 & Day 5 \\
\hline i-PRF & 6 & $0.695 \pm 0.027$ & $0.793 \pm 0.011$ & $0.942 \pm 0.060$ \\
PRP & 6 & $0.676 \pm 0.049$ & $0.682 \pm 0.009$ & $0.825 \pm 0.037$ \\
Cell control & 6 & $0.537 \pm 0.047$ & $0.603 \pm 0.017$ & $0.690 \pm 0.012$
\end{tabular}

$=$ Number of samples

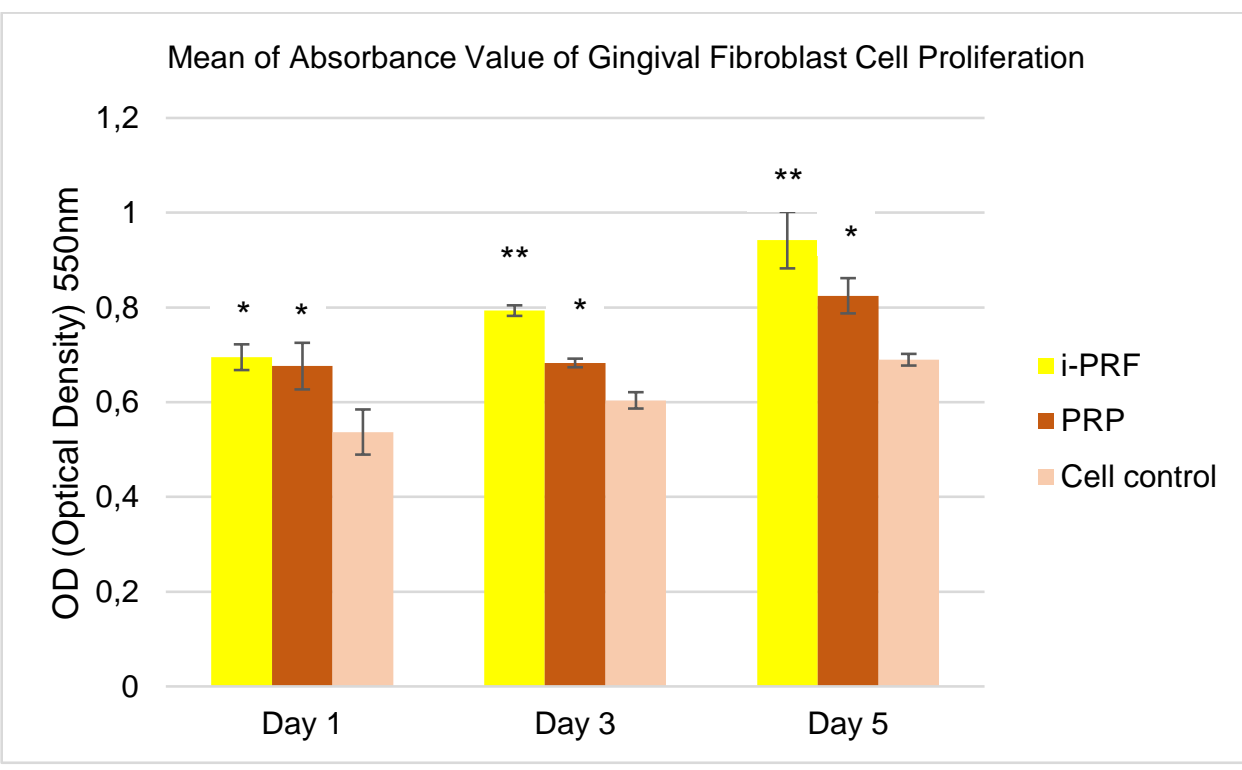

Figure 1. Mean of Absorbance Value of Gingival Fibroblast Cell Proliferation on i-PRF, PRP, and Cell Control on Day 1,3 , and $5\left({ }^{*} p<0.05\right.$ signifies significantly higher than cell control group; ${ }^{* *} p<0.05$ signifies significantly higher than the other group)

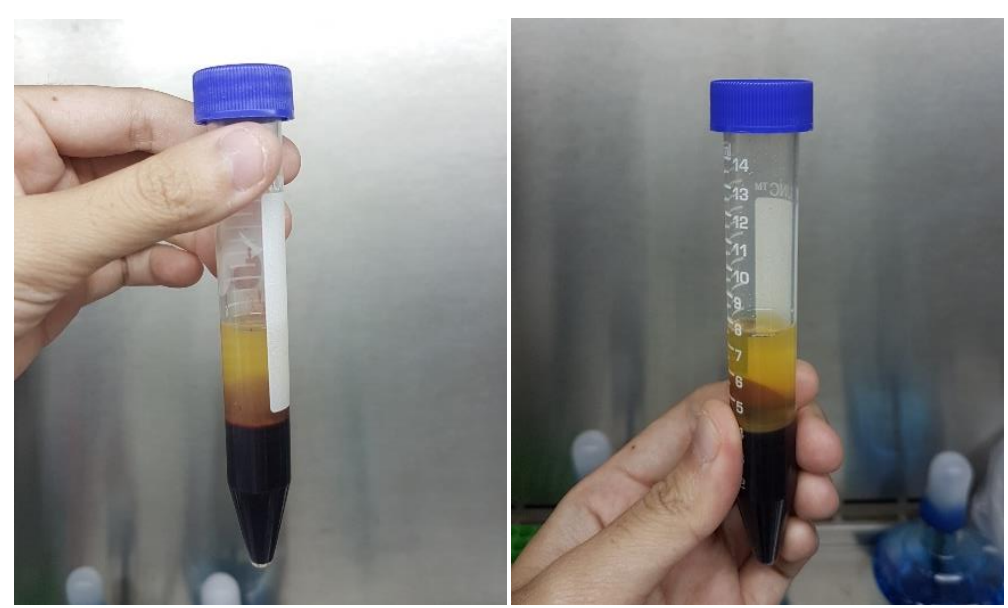

Figure 2. The results of $\mathrm{i}-\mathrm{PRF}$ and PRP centrifugation

\section{DISCUSSION}

The results of statistical tests proved that iPRF and PRP had an effect on the increase of gingival fibroblasts proliferation. Injectable plateletrich fibrin and platelet-rich plasma have similarities 
in their liquid consistency and growth factor properties. Figure 2 showed the results of i-PRF and PRP centrifugation. The difference is in the characteristics of their growth factors. The growth factors of i-PRF are slow release, while the PRP is fast release. i-PRF and PRP have many growth factors, such as VEGF, TGF- $\beta 1$, PDGF, EGF, and IGF-1 which can trigger gingival fibroblast cell proliferation. ${ }^{10,11}$ After 10 days, in i-PRF there were still growth factor releases, whereas PRP had completely dissolved after 10 days. ${ }^{10}$ The growth factor in PRP is secreted in the first hour as much as $95 \% .^{8}$

Preparation of i-PRF, which requires a low centrifugation speed and a short time $(700$ rpm; 3 minutes), will make i-PRF have lots of leukocytes, platelets, and growth factors such as VEGF, TGF$\beta 1$, and PDGF. These growth factors are potent stimulators in gingival fibroblast cell proliferation. ${ }^{11}$ The increase in absorbance value of gingival fibroblast cell proliferation was also seen in the PRP group. A five times higher platelet concentration in the PRP will increase the concentration of growth factor. ${ }^{9}$ The proliferation phase begins on the day- 1 after the occurrence of injury and the increase in new cell proliferation is marked on the day-3. ${ }^{4}$ The success of cell proliferation depends on the availability of the correct growth factors. ${ }^{2}$

The highest mean of absorbance value of gingival fibroblast cell proliferation can be found in the i-PRF group. Injectable platelet-rich fibrin contains lots of leukocytes, platelets, and growth factors to promote soft tissue regeneration. ${ }^{18}$ Platelets can produce VEGF, PDGF, and fibrinogen hence may affect the host immune response. Platelets can also secrete fibrin, fibronectin, and vitronectin, that is acts as a matrix for connective tissue and cell migration adhesion molecules. ${ }^{19}$ Fibrinogen, vitronectin, and fibronectin have a heparin-binding domain that functions to bind tightly to growth factor components. ${ }^{11}$

The main factors for periodontal tissue regeneration consist of: progenitor cells, scaffold, and growth factors. ${ }^{2}$ Figure 3 showed injectable platelet-rich fibrin forms a fibrin gel which functions as a three-dimensional matrix and scaffold biological material for a cluster of cells attached to the regenerated tissue area. The scaffold serves as a 'frame' for stable and effective cell proliferation. In addition, fibrin is a growth factor carrier in a controlled release system. ${ }^{20}$

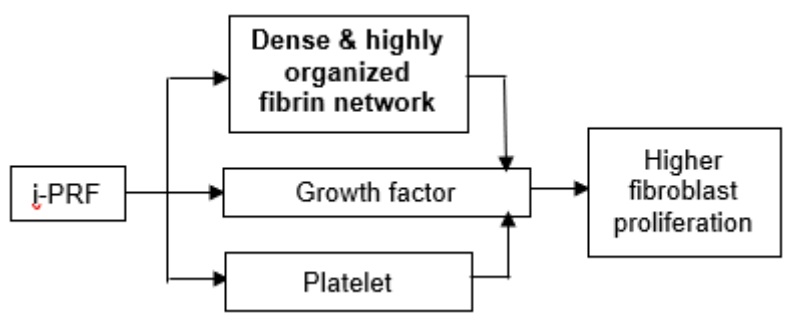

Figure 3. Proliferation mechanism factors of i-PRF

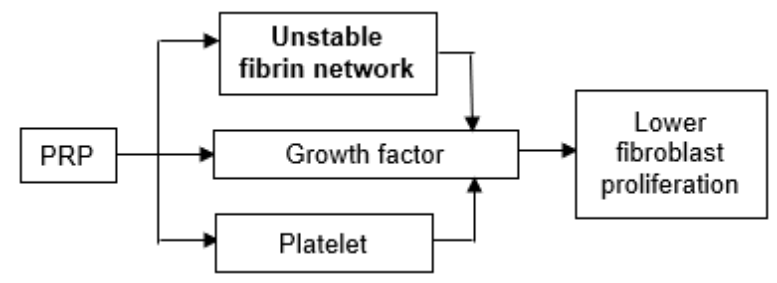

Figure 4. Proliferation mechanism factors of PRP

The lowest mean of absorbance value of gingival fibroblast cell proliferation was found in the PRP group. The release of the growth factor in PRP has been secreted a lot earlier, so it required a faster work time. Growth factor release begins within 10 minutes of PRP preparation, and nearly $95 \%$ is secreted within the first hour. ${ }^{8}$ Figure 4 showed unstable fibrin matrix of PRP leads to low growth factor retention. ${ }^{11}$ Preparation of PRP also uses the anticoagulant EDTA. The use of anticoagulants can suppress the tissue regeneration process. ${ }^{10}$

The cell control group experienced an increase in the absorbance value of gingival 
fibroblast cell proliferation with a significant difference from day-1, 3, and 5 . This is due to the ability of fibroblast cells to proliferate only according to the physiological processes that occur. The content of platelets, leukocytes, and growth factors needs to be enriched to increase the tissue regeneration and wound healing potential. ${ }^{13}$ Therefore, the use of i-PRF is important to increase the gingival fibroblasts proliferation and further study on the fractionation of i-PRF: yellow i-PRF, red $\mathrm{i}-\mathrm{PRF}$, and their combination is needed.

\section{CONCLUSION}

Injectable platelet-rich fibrin and platelet-rich plasma increase the gingival fibroblasts proliferation. Injectable platelet-rich fibrin increases the gingival fibroblasts proliferation higher than platelet-rich plasma.

\section{REFERENCES}

1. Kinane DF, Stathopoulou PG, Papapanou PN. Periodontal Diseases. Nat. Rev. Dis. Primers. 2017; 3(17038): 1-14.

2. Han J, Menicanin D, Gronthos S, Bartold PM. Stem Cells, Tissue Engineering and Periodontal Regeneration. Aust Dent J. 2014; 59(SUPPL.1): 117-30.

3. Menicanin D, Hynes K, Han J, Gronthos S, Bartold PM. Cementum and Periodontal Ligament Regeneration. Adv Exp Med Biol. 2015; 881: 207-36.

4. Smith PC, Martinez C, Martinez J, McCulloch CA. Role of Fibroblasts Populations in Periodontal Wound Healing and Tissue Remodeling. Front Physiol. 2019; 10(270): 1-11.

5. Newman MG, Takei HH, Klokkevold PR, Carranza FA. Anatomy, Structure, and Function of the Periodontium. In: Fiorellini JP, Kim D, Chang $\mathrm{Y}$, editors. Newman and Carranza's Clinical Periodontology Thirteenth Edition. Philadelphia: Elsevier; 2019. p. 209-15.

6. Ardakani MRT, Hajizadeh F, Yadegari Z. Comparison of Attachment and Proliferation of Human Gingival Fibroblasts on Different Collagen Membranes. Ann Maxillofac Surg. 2018; 8(2): 218-23.

7. Suchetha A, Lalwani M, Darshan BM, Sapna N, Bhat D, Sravani K. Growth Factors: Role in Periodontal Regeneration. J Res Med Dent Sci. 2015; 3(3): 166-70.
8. Feigin K, Shope B. Use of Platelet-Rich Plasma and Platelet-Rich Fibrin in Dentistry and Oral Surgery: Introduction and Review of the Literature. J Vet Dent. 2019; 36(2): 109-23.

9. Kobayashi E, Fujioka-Kobayashi M, Sculean A, Chappuis V, Buser D, Schaller B, et al. Effects of Platelet-Rich Plasma (PRP) on Human Gingival Fibroblast, Osteoblast, and Periodontal Cell Ligament Behaviour. BMC Oral Health. 2017; 17(91): 1-10.

10. Miron RJ, Fujioka-Kobayashi M, Hernandez M, Kandalam U, Zhang Y, Ghanaati S, et al. Injectable Platelet-Rich Fibrin (i-PRF): Opportunities in Regenerative Dentistry?. Clin Oral Investig. 2017; 21(8): 2619-27.

11. Thanasrisuebwong $P$, Surarit $R$, Bencharit $S$, Ruangsawasdi N. Influence of Fractionation Methods on Physical and Biological Properties of Injectable Platelet-Rich Fibrin: An Exploratory Study. Int J Mol Sci. 2019; 20(7): 1-10.

12. Wang X, Zhang Y, Choukroun J, Ghanaati S, Miron RJ. Behavior of Gingival Fibroblasts on Titanium Implant Surfaces in Combination with either Injectable-PRF or PRP. Int J Mol Sci. 2017; 18(331): 1-15.

13. Choukroun J, Ghanaati S. Reduction of relative centrifugation force within injectable platelet-rich-fibrin (PRF) concentrates advances patients' own inflammatory cells, platelets and growth factors: the first introduction to the low speed centrifugation concept. Eur J Trauma Emerg Surg. 2018; 44(1): 87- 95.

14. Li X, Yang H, Zhang Z, Yan Z, Lv H, Zhang Y, et al. Platelet-rich fibrin exudate promotes the proliferation and osteogenic differentiation of human periodontal ligament cells in vitro. Mol. Med. Rep. 2018; 18: 4477-85.

15. Nguyen PA, Pham TAV. Effects of platelet-rich plasma on human gingival fibroblast proliferation and migration in vitro. J Appl Oral Sci. 2018; 26: 1-9.

16. Wanichpakorn S, Kedjarune-Laggat $U$. Primary Cell Culture from Human Oral Tissue: Gingival Keratinocytes, Gingival Fibroblasts and Periodontal Ligament Fibroblasts. Songklanakarin J. Sci. Technol. 2010; 32(4): 327-31.

17. Vahabi S, Vaziri S, Torshabi M, Esfahrood ZR. Effects of Plasma Rich in Growth Factor Release and Platelet-Rich Fibrin on Proliferation and Viability of Human Gingival Fibroblasts. J Dent Tehran, 2015; 12(7): 50412.

18. Izol BS, Uner DD. A New Approach for Root Surface Biomodification Using Injectable Platelet-Rich Fibrin (I-PRF). Med Sci Monit. 2019; 25: 4744-50. 
31 THE INFLUENCE BETWEEN INJECTABLE PLATELET-RICH FIBRIN AND PLATELET-RICH PLASMA TOWARDS GINGIVAL FIBROBLAST CELL PROLIFERATION

19. Chandran P, Sivadas A. Platelet-Rich Fibrin: Its Role in Periodontal Regeneration, Saudi J Dent Res. 2014; 5(2): 117-22.

20. Varela HA, Souza JCM, Nascimento RM, Araujo RF, Vasconcelos RC, Cavalcante RS, et al. Injectable Platelet Rich Fibrin: Cell Content, Morphological, and Protein Characterization. Clin Oral Investig. 2019; 23(3): 1309-18. 